\title{
Research Article \\ Can Drag Force Suppress Fermi Acceleration in a Bouncer Model?
}

\author{
Francys Andrews de Souza, ${ }^{1}$ Lucas Eduardo Azevedo Simões, ${ }^{1}$ \\ Mário Roberto da Silva, ${ }^{2}$ and Edson D. Leonel ${ }^{2}$ \\ ${ }^{1}$ Departamento de Matemática, Instituto de Geociências e Ciências Exatas, Universidade Estadual Paulista, \\ Avenida 24A, 1515, Bela Vista, 13506-900 Rio Claro, SP, Brazil \\ ${ }^{2}$ Departamento de Estatística, Matemática Aplicada e Computação, Universidade Estadual Paulista, \\ Avenida 24A, 1515, Bela Vista, 13506-900 Rio Claro, SP, Brazil
}

Correspondence should be addressed to Edson D. Leonel, edleonel@rc.unesp.br

Received 14 April 2009; Accepted 20 July 2009

Recommended by Alexander Loskutov

Some dynamical properties for a bouncer model-a classical particle of mass $m$ falling in the presence of a constant gravitational field $g$ and hitting elastically a periodically moving wall-in the presence of drag force that is assumed to be proportional to the particle's velocity are studied. The dynamics of the model is described in terms of a two-dimensional nonlinear mapping obtained via solution of the second Newton's law of motion. We characterize the behavior of the average velocity of the particle as function of the control parameters as well as the time. Our results show that the average velocity starts growing at first and then bends towards a regime of constant value, thus confirming that the introduction of drag force is a sufficient condition to suppress Fermi acceleration in the model.

Copyright (C) 2009 Francys Andrews de Souza et al. This is an open access article distributed under the Creative Commons Attribution License, which permits unrestricted use, distribution, and reproduction in any medium, provided the original work is properly cited.

\section{Introduction}

The bouncer model consists basically of a classical particle of mass $m$ that moves under the presence of a gravitational field $g$ and suffers elastic collisions with an infinitely heavy and periodically moving wall. It is well known in literature [1-3] that, depending on both the combination of control parameters and initial conditions, the particle can accumulate, along the orbit, large values of energy thus exhibiting a phenomenon called as Fermi acceleration $[4]$.

The bouncer model has been studied along last decades under different versions $[5,6]$ and considering several approaches including theoretical and experimental. For the theoretical approach, results like fixed point stability [7, 8], manifold crossings [9], period doubling cascades, and fully chaotic behavior were discussed [10] both as function of the 
initial conditions and control parameters. For the case of experimental approach, many results have been obtained considering the presence of inelastic collisions [11-13] including the observance of locking phenomenon.

There exist many situations where one can observe Fermi acceleration in onedimensional models. One of them is assuming that the perturbation is no longer periodic but rather stochastic [14]. This perturbation leads to a growth in the average velocity according to square root of the number of collisions of the particle with the randomly moving wall. It is more often to be studied, in such models, conditions where Fermi acceleration (FA) is feasible. Our approach in this paper is rather different. Instead of looking at conditions to produce FA, we will consider a condition to suppress such a phenomenon. Although many results are known in literature concerning the bouncer model, our approach is original in the sense we look at conditions to break down the unlimited energy growth.

In this paper, we consider the bouncer model with the particle experiencing a drag force that is proportional to its velocity. As usual in literature, we have described the model in terms of a two-dimensional nonlinear mapping for the variables velocity of the particle and time at the instant of the collisions with the moving wall. The mapping was obtained by analytical solution of Newton's law of motion. We show that the dissipation suppresses the phenomenon of Fermi acceleration.

The organization of the paper is as follows. In Section 2 we describe all the details used for the mapping construction. Section 3 presents and discusses our numerical results while final remarks and conclusions are drawn in Section 4.

\section{The Model and the Mapping}

In this section we describe with full details the model we are considering and the procedure used to obtain the nonlinear mapping. The system consists of a classical particle of mass $m$ which experiences a drag force that is proportional and contrary to the particle's velocity. The particle is suffering the action of a constant gravitational field $g$, and it collides elastically a time varying wall whose equation of motion is given by $y_{w}(t)=\epsilon \cos (\omega t)$. Here $\epsilon$ is the amplitude of oscillation and $\omega$ denotes the angular frequency of the wall. As it is so common in literature [15-18] the dynamics of the model is described in terms of a two-dimensional mapping $T\left(v_{n}, t_{n}\right)=\left(v_{n+1}, t_{n+1}\right)$ where $v_{n}$ and $t_{n}$ denote the posthit velocity of the particle and the instant of the $n$th collision with the moving wall, respectively.

To obtain the mapping that fully describes the dynamics of the system, we must first write down Newton's law of motion, which is given by $m d \vec{v} / d t=\overrightarrow{m g}-\eta^{\prime} \vec{v}$ where positive $\eta^{\prime}$ is the coefficient of viscosity. Defining $\eta=\eta^{\prime} / m$ we can rewrite the differential equation in scalar form as $d v / d t=-g-\eta v$ where the ascendent motion has positive $v$, and $g$ is the modulus of gravitational acceleration. Integrating with respect to the time this equation assuming that at the time $t=t_{n}$ which is the time that the particle hits the moving wall, the particle is at the position $y_{p}\left(t_{n}\right)=\epsilon \cos \left(\omega t_{n}\right)$ with initial velocity $v\left(t_{n}\right)=v_{n}>0$, and then we find that

$$
v(t)=\left[v_{n}+\frac{g}{\eta}\right] e^{-\eta\left(t-t_{n}\right)}-\frac{g}{\eta}, \quad t \geq t_{n}
$$

Since the expression of the velocity of the particle is known, we must integrate with respect to the time again the relation $d y(t) / d t=v(t)$. Then we have that the position of the 
particle is given by

$$
\begin{aligned}
y_{p}(t)= & \epsilon \cos \left(\omega t_{n}\right)+\frac{1}{\eta}\left(v_{n}+\frac{g}{\eta}\right)\left(1-e^{-\eta\left(t-t_{n}\right)}\right) \\
& -\frac{g}{\eta}\left(t-t_{n}\right), \quad t \geq t_{n} .
\end{aligned}
$$

To find the instant of the collisions of the particle with the moving wall, we must take into account two different kinds of collisions that can occur, namely, (1) successive collisions-those collisions that the particle experiences coming from inside the collision zone $y \in[-\epsilon, \epsilon] ;(2)$ indirect collisions-those collisions where the particle comes from outside the collision zone.

Let us discuss the equations of the mapping for case (1) first. Matching the condition $y_{p}\left(t_{c}\right)=y_{w}\left(t_{c}\right)$ we obtain the instant of the collision $\left(t_{c}=t_{n+1}\right)$ of the particle with the moving wall. Such condition gives rise to the following transcendental equation:

$$
\begin{aligned}
0= & G\left(t_{c}\right)=\epsilon \cos \left[\omega\left(t_{n}+\Delta t_{c}\right)\right]-\epsilon \cos \left(\omega t_{n}\right)+\frac{g}{\eta} \Delta t_{c} \\
& -\frac{1}{\eta}\left(v_{n}+\frac{g}{\eta}\right)\left(1-e^{-\eta \Delta t_{c}}\right),
\end{aligned}
$$

where the interval of collision $\left(\Delta t_{c}=t_{c}-t_{n}\right) \in(0,2 \pi / \omega]$, while exist, is the smallest solution of the equation $G\left(t_{c}\right)=0$ along $\Delta t_{c} \in(0,2 \pi / \omega]$. At the instant of the collision, we assume a noninertial system in which the moving wall is at rest, then the exchange of momentum can be obtained. Finally we return to the original inertial system, leading to a mapping of the type

$$
T_{M}:\left\{\begin{array}{l}
v_{n+1}=-\left[v_{n} e^{-\eta \Delta t_{c}}+\frac{g}{\eta}\left(e^{-\eta \Delta t_{c}}-1\right)\right]-2 \epsilon \omega \sin \left(\omega t_{n+1}\right) \\
t_{n+1}=t_{n}+\Delta t_{c}
\end{array}\right.
$$

We now discuss case (2), that is, indirect collisions. In the approach we have considered there are three steps to obtain the time the particle spent between collisions in the bouncer model. The first one is the elapsed time from the last collision up to the maximum height where the particle acquires null velocity. The second one is the time the particle spent from the maximum height up to the entrance of the collision zone. Finally the third time is the time the particle spent from the entrance of the collision zone up to experiences a collision with the moving wall again. With these steps in mind, we must first find the maximum height that the particle reaches, which is obtained by considering the case $v\left(t_{u}\right)=0$ where $\Delta t_{u}=t_{u}-t_{n}$ corresponds to the time the particle spent from the last collision up to the maximum height. It is given by $\Delta t_{u}=-\ln \left[g /\left(\eta v_{n}+g\right)\right] / \eta$. At this time, the position of the particle is

$$
y_{p}\left(t_{u}\right)=h_{\max }=\epsilon \cos \left(\omega t_{n}\right)+\frac{v_{n}}{\eta}+\frac{g}{\eta^{2}} \ln \left(\frac{g}{\eta v_{n}+g}\right) .
$$


From this maximum height $\left(h_{\max }\right)$, the expression that describes the particle's position in the downwards motion is given by

$$
\begin{aligned}
y_{p}(t)= & \epsilon \cos \left(\omega t_{n}\right)+\frac{v_{n}}{\eta}+\frac{g}{\eta^{2}} \ln \left(\frac{g}{\eta v_{n}+g}\right) \\
& +\frac{g}{\eta^{2}}\left(1-e^{-\eta\left(t-t_{u}\right)}\right)-\frac{g}{\eta}\left(t-t_{u}\right), \quad \text { if } t \geq t_{u} .
\end{aligned}
$$

The next step is to obtain the time the particle spends until the entrance of the collision zone. The instant $t_{d}$ is obtained by matching the condition $y_{p}\left(t_{d}\right)=\epsilon$, leading then to the following transcendental function $Z(t)=y_{p}(t)-\epsilon$ that must be solved:

$$
\begin{aligned}
0= & Z\left(t_{d}\right)=\epsilon \cos \left(\omega t_{n}\right)+\frac{v_{n}}{\eta}+\frac{g}{\eta^{2}} \ln \left(\frac{g}{\eta v_{n}+g}\right) \\
& +\frac{g}{\eta^{2}}\left(1-e^{-\eta \Delta t_{d}}\right)-\frac{g}{\eta} \Delta t_{d}-\epsilon,
\end{aligned}
$$

where $\Delta t_{d}=t_{d}-t_{u}$. Since we obtain the solution of the above equation, then we found that the velocity of the particle at the entrance of the collision zone is $v_{e}=v\left(t_{d}\right)$, that is written as $v_{e}=g / \eta\left(\exp \left(-\eta \Delta t_{d}\right)-1\right)$. From there, the expression of the particle velocity is given by

$$
v(t)=e^{-\eta\left(t-t_{d}\right)}\left(v_{e}+\frac{g}{\eta}\right)-\frac{g}{\eta} \quad \text { if } t \geq t_{d}
$$

To find the instant of the collision of the particle with the moving wall, we must solve the following equation $F\left(t_{c}\right)=y_{w}\left(t_{c}\right)-y_{p}\left(t_{c}\right)=0$ where $y_{w}(t)$ corresponds to the moving wall position and $y_{p}(t)$ is the particle position obtained by integration of $(2.8)$. Then we found that

$$
\begin{aligned}
0= & F\left(t_{c}\right)=\frac{1}{\eta}\left(v_{e}+\frac{g}{\eta}\right)-\frac{g}{\eta} \Delta t_{c}-\frac{1}{\eta}\left(v_{e}+\frac{g}{\eta}\right) e^{-\eta \Delta t_{c}} \\
& -\epsilon \cos \left(\omega\left(t_{n}+\Delta t_{u}+\Delta t_{d}+\Delta t_{c}\right)\right)+\epsilon,
\end{aligned}
$$

where $\Delta t_{c}=t_{c}-t_{d}$ is the smallest positive root of the above equation for the interval $\Delta t_{c} \in$ $[0,2 \pi / \omega)$. Thus, in the instant of the collision we have

$$
T_{I}=\left\{\begin{array}{l}
v_{n+1}=-\left[e^{-\eta \Delta t_{c}}\left(v_{e}+\frac{g}{\eta}\right)-\frac{g}{\eta}\right]-2 \epsilon \omega \sin \left(\omega t_{n+1}\right), \\
t_{n+1}=t_{n}+\Delta t_{u}+\Delta t_{d}+\Delta t_{c},
\end{array}\right.
$$

where $\Delta t_{c}$ is obtained from the smallest solution of $F\left(t_{c}\right)=0$.

We can see that there is a relatively large number of control parameters, 4 in total, namely, $\epsilon, \eta, \omega, g$ and that the dynamics of the system does not depend on all of them. It is convenient to define dimensionless and more appropriate variables. Thus we define $V=$ $\left(v_{n} \omega\right) / g, \delta=\omega / \eta, a=\left(\epsilon \omega^{2}\right) / g$, and measure the time in terms of the number of oscillations 
of the moving wall, $\phi=\omega t$. With this new set of variables, the mapping that describes the dynamics of the model is given by

$$
T=\left\{\begin{array}{l}
V_{n+1}=-\left[\left(V_{n}^{*}+\delta\right) e^{-\Delta \phi_{c} / \delta}-\delta\right]-2 a \sin \left(\phi_{n+1}\right) \\
\phi_{n+1}=\left[\phi_{n}+\Delta \phi\right] \quad \bmod (2 \pi)
\end{array}\right.
$$

where the variables $V_{n}^{*}$ and $\Delta \phi$ depend on the kind of collisions that occurs. For successive collisions we have that $V_{n}^{*}=V_{n}$ and $\Delta \phi=\Delta \phi_{c}$ where $\Delta \phi_{c}$ is obtained as the smallest positive solution of $G\left(\phi_{c}\right)=0$ for $\Delta \phi_{c} \in(0,2 \pi]$, with

$$
\begin{aligned}
0= & G\left(\phi_{c}\right)=a \cos \left(\phi_{n}\right)+\delta\left(V_{n}+\delta\right)\left(1-e^{-\Delta \phi_{c} / \delta}\right) \\
& -\delta \Delta \phi_{c}-a \cos \left(\phi_{n}+\Delta \phi_{c}\right) .
\end{aligned}
$$

For the case of indirect collisions, we have that $V_{n}^{*}=V_{e}$ where $V_{e}=\delta\left[\exp \left(-\Delta \phi_{d} / \delta\right)-1\right]$ and $\Delta \phi=\Delta \phi_{u}+\Delta \phi_{d}+\Delta \phi_{c}$ with $\Delta \phi_{u}=-\delta \ln \left(\delta /\left(V_{n}+\delta\right)\right)$ corresponding to the upward elapsed time, and $\Delta \phi_{d}$ is obtained as the smallest positive root of $Z\left(\phi_{d}\right)=0$ with

$$
\begin{aligned}
0= & Z\left(\phi_{d}\right)=a \cos \left(\phi_{n}\right)+\delta V_{n}+\delta^{2} \ln \left(\frac{\delta}{V_{n}+\delta}\right) \\
& +\delta^{2}\left(1-e^{-\Delta \phi_{d} / \delta}\right)-\delta \Delta \phi_{d}-a
\end{aligned}
$$

where $\Delta \phi_{d}$ is the elapsed time along the downward direction until the entrance of the collision zone. Finally $\Delta \phi_{c}$ is the smallest positive solution of $F\left(\phi_{c}\right)=0$, with

$$
\begin{aligned}
0= & F\left(\phi_{c}\right)=a+\delta\left(V_{e}+\delta\right)-\delta \Delta \phi_{c}-\delta\left(V_{e}+\delta\right) e^{-\Delta \phi_{c} / \delta} \\
& -a \cos \left(\phi_{n}+\Delta \phi_{u}+\Delta \phi_{d}+\Delta \phi_{c}\right) .
\end{aligned}
$$

After some straightforward algebra we found that the determinant of the Jacobian matrix is written as

$$
\operatorname{det}\left(\begin{array}{cc}
\frac{\partial V_{n+1}}{\partial V_{n}} & \frac{\partial V_{n+1}}{\partial \phi_{n}} \\
\frac{\partial \phi_{n+1}}{\partial V_{n}} & \frac{\partial \phi_{n+1}}{\partial \phi_{n}}
\end{array}\right)=A\left(\frac{V_{n}+a \sin \left(\phi_{n}\right)}{V_{n+1}+a \sin \left(\phi_{n+1}\right)}\right)
$$

where $A$ depends on the kind of collisions. For multiple collisions $A=\exp \left(-\phi_{c} / \delta\right)$ and for indirect collisions $A=\delta \exp \left(-\left[\phi_{c}+\phi_{d}\right] / \delta\right) /\left(V_{n}+\delta\right)$. We note that when $\delta \rightarrow \infty$, which is equivalent to $\eta \rightarrow 0$, then $A \rightarrow 1$ and therefore all results for the non dissipative case are recovered. 


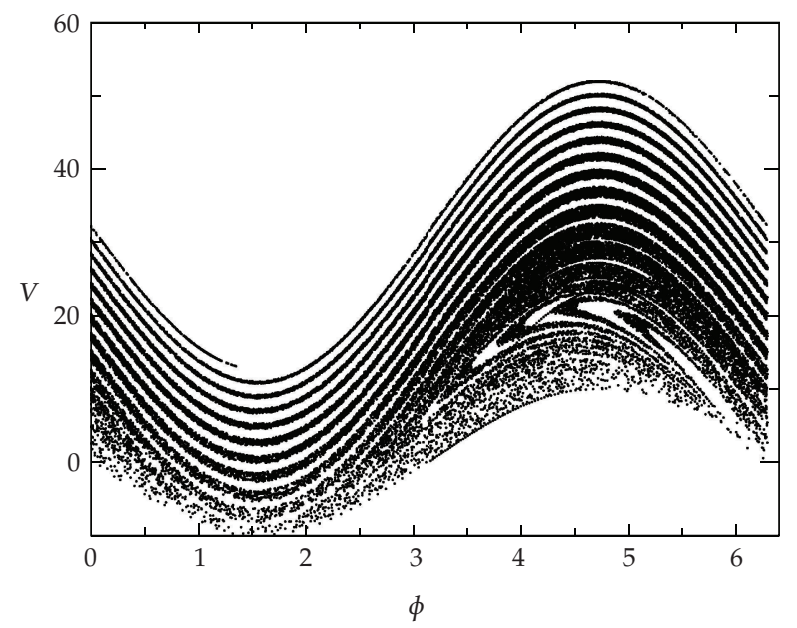

(a)

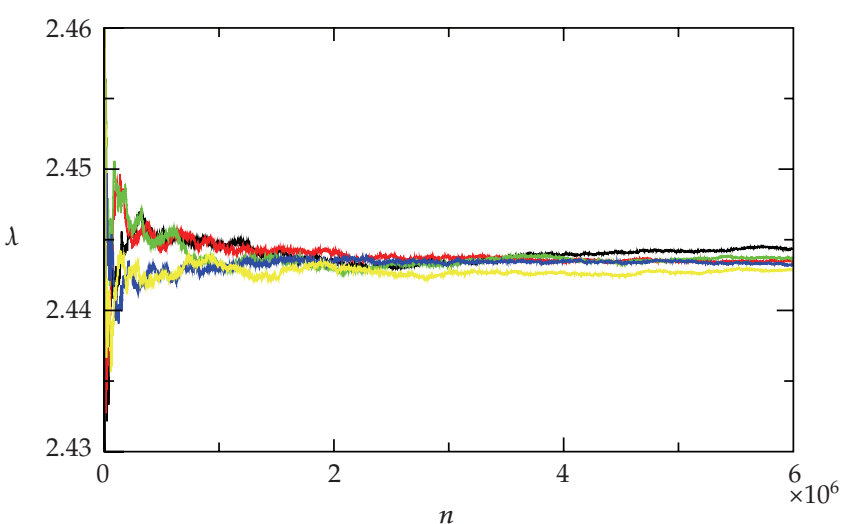

(b)

Figure 1: (a) represents a chaotic attractor for the dissipative bouncer model. The control parameters used were $\delta=60$ and $a=10$. (b) corresponds to the convergence of the positive Lyapunov exponent for five different initial conditions for the same control parameters used in (a).

\section{Numerical Results}

In this section we will present and discuss our numerical results for the dissipative bouncer model. It is shown in Figure 1(a) the behavior of a chaotic attractor in phase space for the control parameters $\delta=60$ and $a=10$. To confirm that the orbit shown in Figure 1(a) is really chaotic, we should then evaluate the Lyapunov exponent and check whether the larger value is positive [19]. It is well known in literature that the Lyapunov exponent is a good tool that can be used to quantify the sensitivity to the initial conditions. They are defined as

$$
\lambda_{j}=\lim _{n \rightarrow \infty} \frac{1}{n} \sum_{i=1}^{n} \ln \left|\Lambda_{j}^{i}\right|, \quad j=1,2
$$




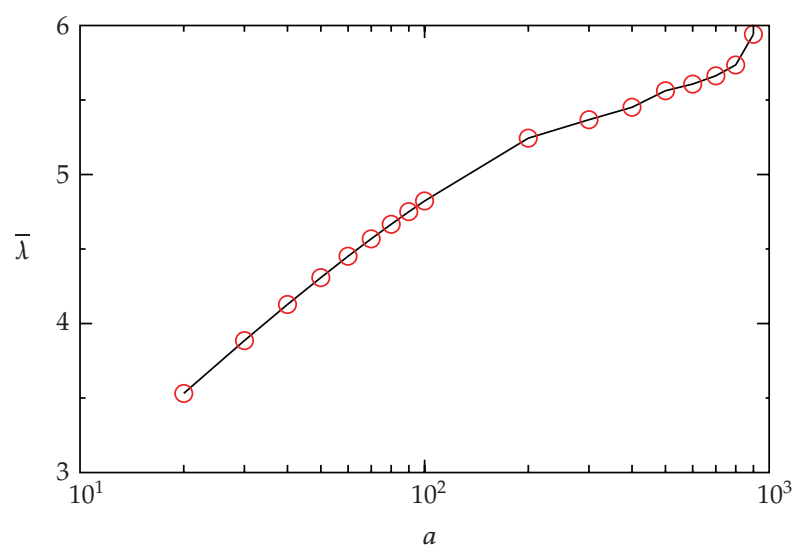

(a)

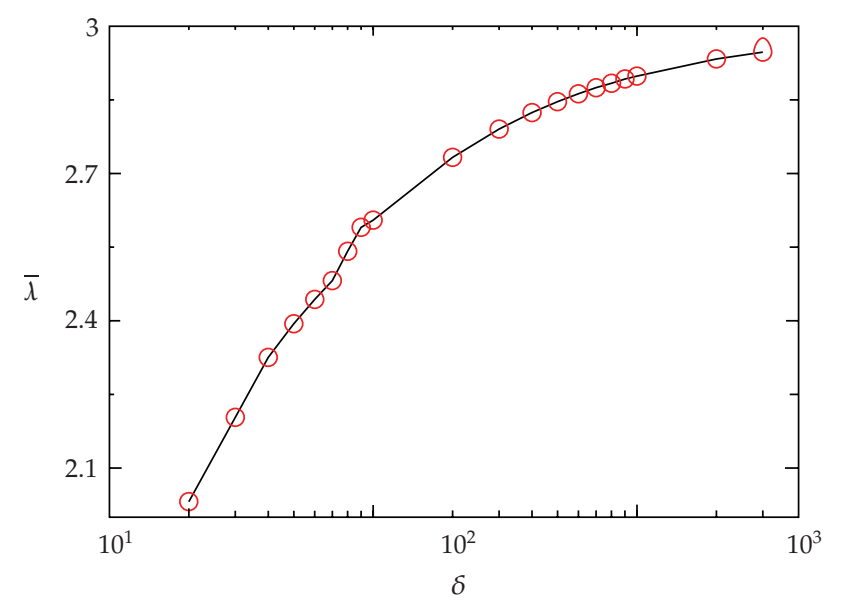

(b)

Figure 2: Positive Lyapunov exponent as function of the control parameter: (a) $a$ and (b) $\delta$.

where $\Lambda_{j}^{i}$ are the eigenvalues of the matrix $M=\prod_{i=1}^{n} J_{i}\left(V_{i}, \phi_{i}\right)$ with $J_{i}$ representing the Jacobian matrix of the mapping. In the appendix we show all the expressions of the Jacobian matrix for both multiple and indirect collisions.

After implementing the algorithm in a computer code, we found that the Lyapunov exponent for the attractor shown in Figure 1(a) is positive as can be seen in Figure 1(b). We have evolved in time five different initial conditions for the control parameters $\delta=60$ and $a=10$ and saw that both converge for long time for a constant value of $\bar{\lambda}=2.443 \pm 0.003$.

We have also obtained the behavior of the positive Lyapunov exponent for the dissipative bouncer model as a function of the control parameters $a$ and $\delta$, as we can see in Figures 2(a) and 2(b). Each point in Figure 2 was obtained as an average of five different initial conditions along the chaotic attractor and evolved up to $6 \cdot 10^{6}$ collisions of the particle with the moving wall. We can see that the positive Lyapunov exponent grows as a function of $a$ as well as a function of $\delta$.

Let us now discuss the behavior of the average velocity for chaotic orbits. To obtain the average velocity we consider two different kinds of averages: (1) average over the orbit 


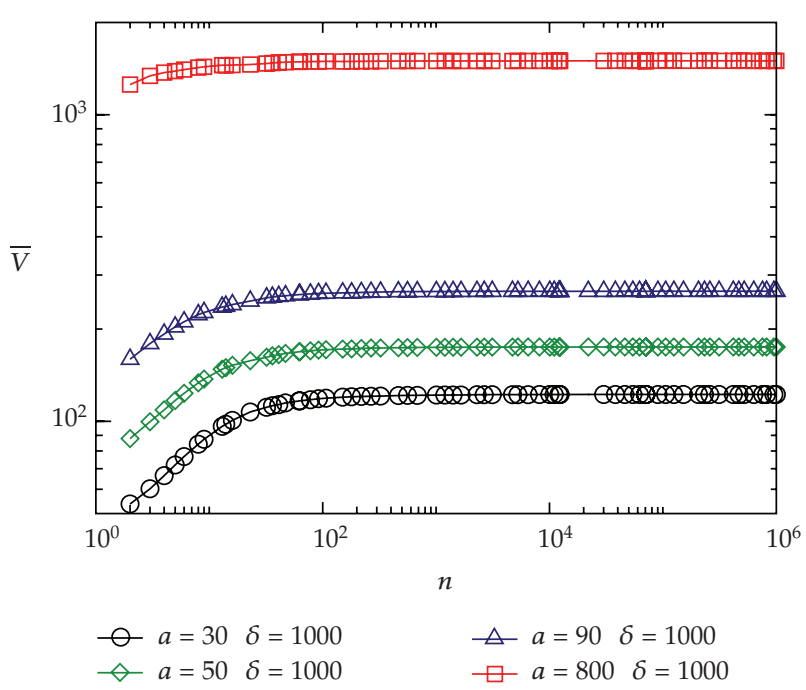

(a)

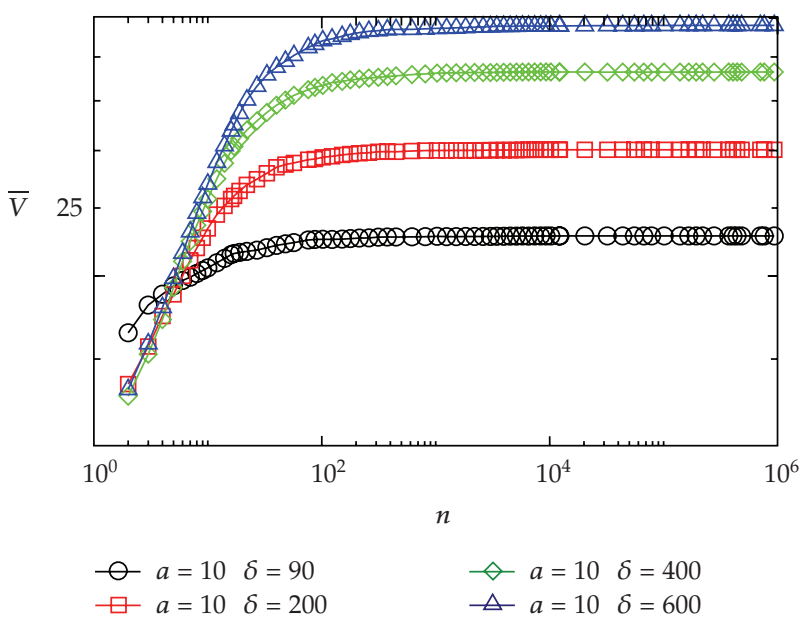

(b)

Figure 3: Behavior of $\bar{V} \times n$ for different control parameter, as shown in the figure.

(sometimes also called as Birkoff's average) and (2) average over an ensemble of initial conditions. The average over the orbit is obtained by evolving for a long time a single initial condition so that we have

$$
V_{i}=\frac{1}{n} \sum_{j=1}^{n} V_{i, j}
$$

where $j$ corresponds to the number of collisions and $i$ corresponds to a sample in an ensemble of $M$ different initial conditions. After that, we can average over different initial conditions 
leading to

$$
\bar{V}=\frac{1}{M} \sum_{i=1}^{M} V_{i}
$$

where $M$ is the number of different initial conditions used. It is then shown in Figures 3(a) and 3(b) the behavior of $\bar{V} \times n$ for different control parameters. We can see that the velocity grows at first and then approaches a regime of saturation for long enough time (similar results for nondissipative dynamics have also been observed [20-22]). We consider now the behavior of the velocity for long time, thus corresponding to the constant plateau. Such plateaus are clear evidence that the phenomenon of Fermi acceleration has been suppressed [14].

After a careful analysis of Figures 3(a) and 3(b) we can see that as both the control parameters rise, the value of the average velocity for long time, we call it now as $\bar{V}_{\text {sat, also }}$ rises. Such a behavior allows us to suppose that

$$
\bar{V}_{\text {sat }} \propto \delta^{\alpha_{1}} a^{\alpha_{2}}
$$

where $\alpha_{1}$ and $\alpha_{2}$ are critical exponents that must be obtained.

If we obtain the behavior of $\bar{V}_{\text {sat }}$ as function of both $a$ and $\delta$, we can then obtain the critical exponents $\alpha_{1}$ and $\alpha_{2}$. It is shown in Figures 4(a) and 4(b) the behavior of $\bar{V}_{\text {sat }}$ as a function of the control parameter $a$ and $\delta$. After adjusting a power law fitting, we obtain that the critical exponents are $\alpha_{1}=0.299 \pm 0.003$ and $\alpha_{2}=(0.747 \pm 0.007) \cong 3 / 4$.

Let us now discuss the consequences of the results obtained above. If we increase the value of the control parameter $a$, it corresponds to enlarge the ratio of accelerations, then allowing the particle to acquire more energy upon collision with the moving wall. This result is then confirmed since the critical exponent obtained was $\alpha_{2}=0.747 \pm 0.007$. Similar result can be obtained as function of the control parameter $\delta$. However, we must emphasize that as the control parameter $\delta$ rises, the straight of the dissipation decreases since $\delta \propto 1 / \eta$. So in the limit of very large values of $\delta$, almost no dissipation is present. For such a regime, it is then expected that Fermi acceleration should holds. Our results confirm such a supposition since the critical exponent $\alpha_{1}=0.299 \pm 0.003$ leading then to the phenomenon of Fermi acceleration for the regime of $\delta \rightarrow \infty$. However for finite values of $\delta$, suppression of Fermi acceleration is observed. This result enlarges the condition where FA is suppressed since it is already known [14] that inelastic collision also suppress Fermi acceleration.

\section{Conclusions}

We have studied the bouncer model with the presence of a drag force that is proportional to particle's velocity. The model was described by using a two-dimensional nonlinear map, obtained by the solution of a Newton's law of motion. We show that chaotic attractors are present in the system and we have characterized them by use of the positive Lyapunov exponent. We have shown that the introduction of a drag force leads the particle to experience a limited energy gain. Then as to answer the question proposed in the title, the introduction of a drag force proportional to the particle's velocity can indeed be assumed as a mechanism to suppress Fermi acceleration. Such suppression is topologically demostrated by the determinant, of the Jacobian matrix. Since the pre-factor A in the determinant of the 


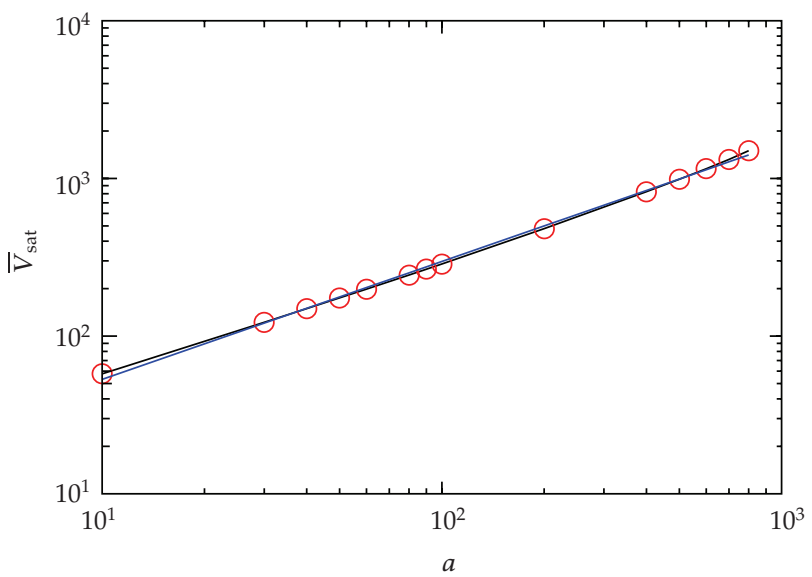

(a)

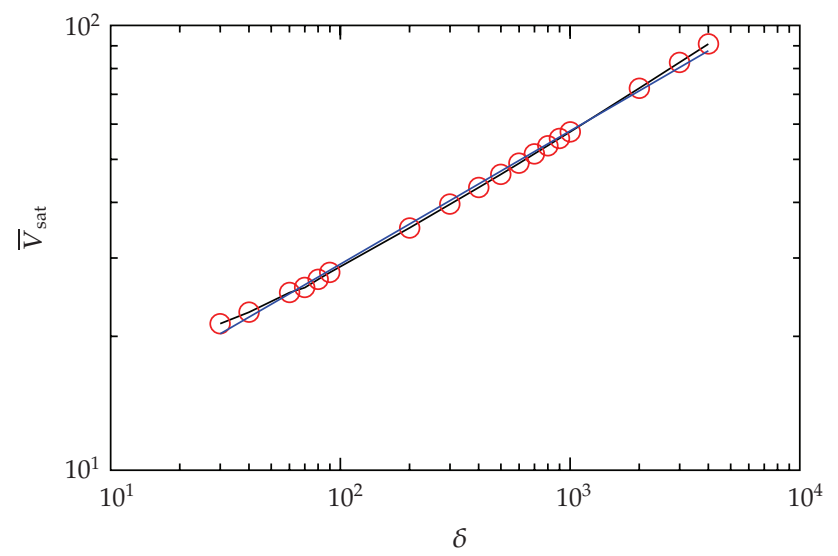

(b)

Figure 4: Behavior of $\bar{V}_{\text {sat }}$ as function of (a) $a$ and (b) $\delta$. After a power law fitting we obtain that $\alpha_{1}=$ $0.299 \pm 0.003$ and $\alpha_{2}=(0.747 \pm 0.007) \cong 3 / 4$.

Jacobian matrix is less than the unity, attractors are present in the phase space; thus unlimited energy growth is not feasible anymore.

\section{Appendix}

We present in this appendix all the expressions of the partial derivatives of the Jacobian matrix. We start with the expressions obtained for the multiple collisions

$$
\begin{gathered}
\frac{\partial V_{n+1}}{\partial V_{n}}=e^{-\Delta \phi_{c} / \delta}\left[\frac{\partial \phi_{c}}{\partial V_{n}}\left(1+\frac{V_{n}}{\delta}\right)-1\right]-2 a \cos \left(\phi_{n+1}\right) \frac{\partial \phi_{n+1}}{\partial V_{n}}, \\
\frac{\partial V_{n+1}}{\partial \phi_{n}}=e^{-\Delta \phi_{c} / \delta} \frac{\partial \Delta \phi_{c}}{\partial \phi_{n}}\left(1+\frac{V_{n}}{\delta}\right)-2 a \cos \left(\phi_{n+1}\right) \frac{\partial \phi_{n+1}}{\partial \phi_{n}},
\end{gathered}
$$


Mathematical Problems in Engineering

$$
\begin{gathered}
\frac{\partial \phi_{n+1}}{\partial V_{n}}=\frac{\partial \phi_{c}}{\partial V_{n}}, \\
\frac{\partial \phi_{n+1}}{\partial \phi_{n}}=\frac{\partial \phi_{c}}{\partial \phi_{n}}+1,
\end{gathered}
$$

where $\partial \phi_{c} / \partial V_{n}$ and $\partial \phi_{c} / \partial \phi_{n}$ are obtained, respectively, via implicit derivative of $G\left(\Delta \phi_{c}\right)$ with respect to $V_{n}$ and $\phi_{n}$. Their expressions are

$$
\begin{aligned}
\frac{\partial \phi_{c}}{\partial V_{n}} & =\frac{\delta\left(e^{-\Delta \phi_{c} / \delta}-1\right)}{e^{-\Delta \phi_{c} / \delta}\left(V_{n}+\delta\right)-\delta+a \sin \left(\phi_{n+1}\right)} \\
\frac{\partial \phi_{c}}{\partial \phi_{n}} & =\frac{a \sin \left(\phi_{n}\right)-a \sin \left(\phi_{n+1}\right)}{\left(V_{n}+\delta\right) e^{-\Delta \phi_{c} / \delta}-\delta+a \sin \left(\phi_{n}+1\right)} .
\end{aligned}
$$

We now obtain the expression of the Jacobian terms for the indirect collisions

$$
\begin{gathered}
\frac{\partial V_{n+1}}{\partial V_{n}}=e^{-\Delta \phi_{c} / \delta}\left[\frac{\partial \Delta \phi_{c}}{\partial V_{n}}\left(1+\frac{V_{e}}{\delta}\right)-\frac{\partial V_{e}}{\partial V_{n}}\right]-2 a \cos \left(\phi_{n+1}\right) \frac{\partial \phi_{n+1}}{\partial V_{n}} \\
\frac{\partial V_{n+1}}{\partial \phi_{n}}=e^{-\Delta \phi_{c} / \delta}\left[\frac{\partial \Delta \phi_{c}}{\partial \phi_{n}}\left(1+\frac{V_{e}}{\delta}\right)-\frac{\partial V_{e}}{\partial \phi_{n}}\right]-2 a \cos \left(\phi_{n+1}\right) \frac{\partial \phi_{n+1}}{\partial \phi_{n}} \\
\frac{\partial \phi_{n+1}}{\partial V_{n}}=\frac{\partial \Delta \phi_{u}}{\partial V_{n}}+\frac{\partial \Delta \phi_{d}}{\partial V_{n}}+\frac{\partial \Delta \phi_{c}}{\partial V_{n}}, \\
\frac{\partial \phi_{n+1}}{\partial \phi_{n}}=1+\frac{\partial \Delta \phi_{d}}{\partial \phi_{n}}+\frac{\partial \Delta \phi_{c}}{\partial \phi_{n}}
\end{gathered}
$$

where $\partial \phi_{d} / \partial V_{n}, \partial \phi_{d} / \partial \phi_{n}, \partial \phi_{c} / \partial V_{n}$, and $\partial \phi_{c} / \partial \phi_{n}$ are obtained by implicit derivative of $Z\left(\Delta \phi_{c}\right)$ and $F\left(\Delta \phi_{c}\right)$ with respect to $V_{n}$ and $\phi_{n}$. Their expressions are

$$
\begin{gathered}
\frac{\partial \Delta \phi_{d}}{\partial V_{n}}=\frac{\partial \Delta \phi_{u} / \partial V_{n}-1}{e^{-\Delta \phi_{d} / \delta}-1}, \\
\frac{\partial \Delta \phi_{d}}{\partial \phi_{n}}=\frac{a \sin \left(\phi_{n}\right)}{\delta\left(e^{-\Delta \phi_{d} / \delta}-1\right)}, \\
\frac{\partial \Delta \phi_{c}}{\partial V_{n}}=\frac{\delta\left(\partial V_{e} / \partial V_{n}\right)\left(1-e^{-\Delta \phi_{c} / \delta}\right)+a \sin \left(\phi_{n+1}\right)\left(\left(\partial \Delta \phi_{u} / \partial V_{n}\right)\left(\partial \Delta \phi_{d} / \partial V_{n}\right)\right)}{\delta-e^{-\Delta \phi_{c} / \delta}\left(V_{e}+\delta\right)-a \sin \left(\phi_{n+1}\right)}, \\
\frac{\partial \Delta \phi_{c}}{\partial \phi_{n}}=\frac{\delta\left(\partial V_{e} / \partial \phi_{n}\right)\left(1-e^{-\Delta \phi_{c} / \delta}\right)+a \sin \left(\phi_{n+1}\right)\left(\partial \Delta \phi_{d} / \partial \phi_{n}+1\right)}{\delta-e^{-\Delta \phi_{c} / \delta}\left(V_{e}+\delta\right)-a \sin \left(\phi_{n+1}\right)}
\end{gathered}
$$


where $\partial \Delta \phi_{u} / \partial V_{n}, \partial V_{e} / \partial V_{n}$, and $\partial V_{e} / \partial \phi_{n}$ are the implicit derivative of $\Delta \phi_{u}$ with respect to $V_{n}$ and the the implicit derivative of $V_{e}$ with respect to $V_{n}$ and $\phi_{n}$, respectively. They are written as

$$
\begin{gathered}
\frac{\partial \Delta \phi_{u}}{\partial V_{n}}=\frac{\delta}{V_{n}+\delta^{\prime}}, \\
\frac{\partial V_{e}}{\partial V_{n}}=-e^{-\Delta \phi_{d} / \delta} \frac{\partial \Delta \phi_{d}}{\partial V_{n}}, \\
\frac{\partial V_{e}}{\partial \phi_{n}}=-e^{-\Delta \phi_{d} / \delta} \frac{\partial \Delta \phi_{d}}{\partial \phi_{n}} .
\end{gathered}
$$

\section{Acknowledgments}

F. A. de Souza and L. E. A. Simões thank FAPESP, Brazilian agency. E. D. Leonel gratefully acknowledges support to CNPq, FAPESP and FUNDUNESP, Brazilian agencies.

\section{References}

[1] A. J. Lichtenberg and M. A. Lieberman, Regular and Chaotic Dynamics, vol. 38 of Applied Mathematical Sciences, Springer, New York, NY, USA, 2nd edition, 1992.

[2] A. J. Lichtenberg, M. A. Lieberman, and R. H. Cohen, "Fermi acceleration revisited," Physica D, vol. 1, no. 3, pp. 291-305, 1980.

[3] E. D. Leonel and P. V. E. McClintock, "A crisis in the dissipative Fermi accelerator model," Journal of Physics A, vol. 38, no. 23, pp. L425-L430, 2005.

[4] E. Fermi, “On the origin of the cosmic radiation," Physical Review, vol. 75, no. 6, pp. 1169-1174, 1949.

[5] A. L. P. Livorati, D. G. Ladeira, and E. D. Leonel, "Scaling investigation of Fermi acceleration on a dissipative bouncer model," Physical Review E, vol. 78, no. 5, Article ID 056205, 12 pages, 2008.

[6] E. D. Leonel and A. L. P. Livorati, "Describing Fermi acceleration with a scaling approach: the bouncer model revisited," Physica A, vol. 387, no. 5-6, pp. 1155-1160, 2008.

[7] P. J. Holmes, "The dynamics of repeated impacts with a sinusoidally vibrating table," Journal of Sound and Vibration, vol. 84, no. 2, pp. 173-189, 1982.

[8] J. J. Barroso, M. V. Carneiro, and E. E. N. Macau, "Bouncing ball problem: stability of the periodic modes," Physical Review E, vol. 79, no. 2, Article ID 026206, 10 pages, 2009.

[9] R. M. Everson, “Chaotic dynamics of a bouncing ball," Physica D, vol. 19, no. 3, pp. 355-383, 1986.

[10] D. G. Ladeira and E. D. Leonel, "Dynamical properties of a dissipative hybrid Fermi-Ulam-bouncer model," Chaos, vol. 17, no. 1, Article ID 013119, 7 pages, 2007.

[11] J. M. Luck and A. Mehta, "Bouncing ball with a finite restitution: chattering, locking, and chaos," Physical Review E, vol. 48, no. 5, pp. 3988-3997, 1993.

[12] G. A. Luna-Acosta, "Regular and chaotic dynamics of the damped Fermi accelerator," Physical Review A, vol. 42, no. 12, pp. 7155-7162, 1990.

[13] M. A. Naylor, P. Sánchez, and M. R. Swift, "Chaotic dynamics of an air-damped bouncing ball," Physical Review E, vol. 66, no. 5, Article ID 057201, 3 pages, 2002.

[14] E. D. Leonel, "Breaking down the Fermi acceleration with inelastic collisions," Journal of Physics A, vol. 40, no. 50, pp. F1077-F1083, 2007.

[15] A. K. Karlis, P. K. Papachristou, F. K. Diakonos, V. Constantoudis, and P. Schmelcher, "Hyperacceleration in a stochastic Fermi-Ulam model," Physical Review Letters, vol. 97, no. 19, Article ID 194102, 2006.

[16] A. K. Karlis, P. K. Papachristou, F. K. Diakonos, V. Constantoudis, and P. Schmelcher, "Fermi acceleration in the randomized driven Lorentz gas and the Fermi-Ulam model," Physical Review E, vol. 76, no. 1, Article ID 016214, 17 pages, 2007.

[17] E. D. Leonel and P. V. E. McClintock, "Effect of a frictional force on the Fermi-Ulam model," Journal of Physics A, vol. 39, no. 37, pp. 11399-11415, 2006. 
[18] E. D. Leonel and M. R. Silva, "A bouncing ball model with two nonlinearities: a prototype for Fermi acceleration," Journal of Physics. A: Mathematical and Theoretical, vol. 41, no. 1, Article ID 015104, 13 pages, 2008.

[19] J.-P. Eckmann and D. Ruelle, "Ergodic theory of chaos and strange attractors," Reviews of Modern Physics, vol. 57, no. 3, part 1, pp. 617-656, 1985.

[20] E. D. Leonel, P. V. E. McClintock, and J. K. L. da Silva, “Fermi-Ulam accelerator model under scaling analysis," Physical Review Letters, vol. 93, no. 1, Article ID 014101, 4 pages, 2004.

[21] D. G. Ladeira and J. K. L. da Silva, "Time-dependent properties of a simplified Fermi-Ulam accelerator model," Physical Review E, vol. 73, no. 2, Article ID 026201, 6 pages, 2006.

[22] J. K. L. Da Silva, D. G. Ladeira, E. D. Leonel, P. V. E. McClintock, and S. O. Kamphorst, "Scaling properties of the fermi-ulam accelerator model," Brazilian Journal of Physics, vol. 36, no. 3 A, pp. 700$707,2006$. 


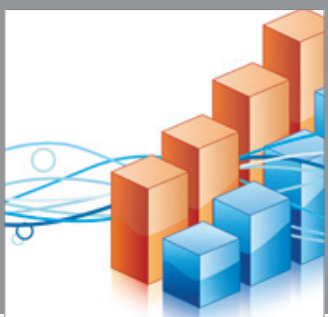

Advances in

Operations Research

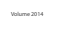

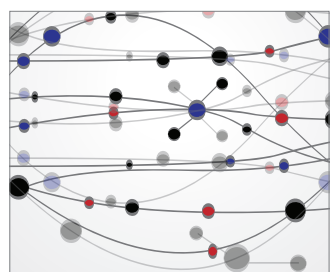

\section{The Scientific} World Journal
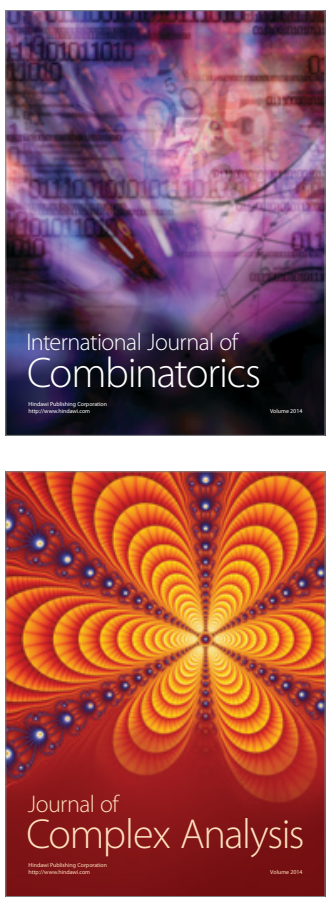

International Journal of

Mathematics and

Mathematical

Sciences
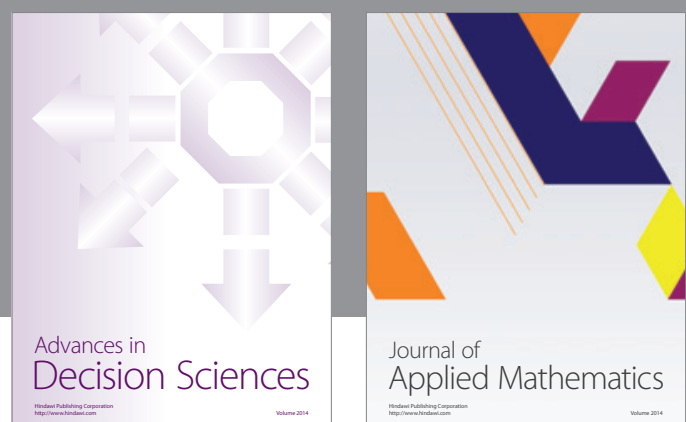

Journal of

Applied Mathematics
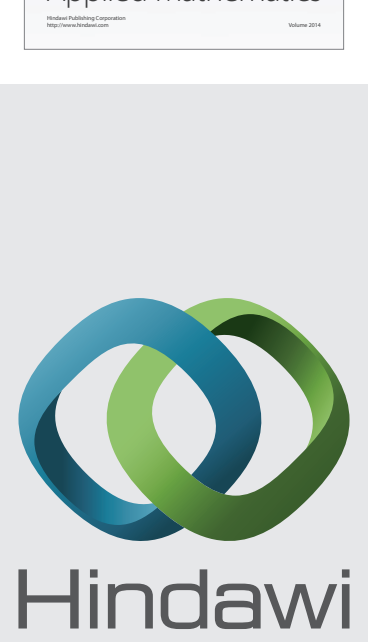

Submit your manuscripts at http://www.hindawi.com
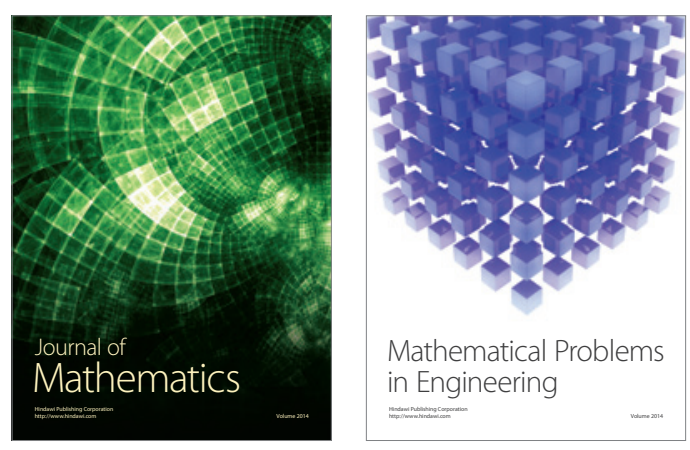

Mathematical Problems in Engineering
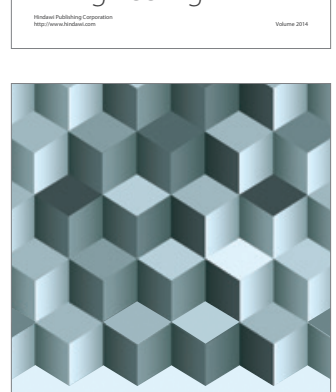

Journal of

Function Spaces
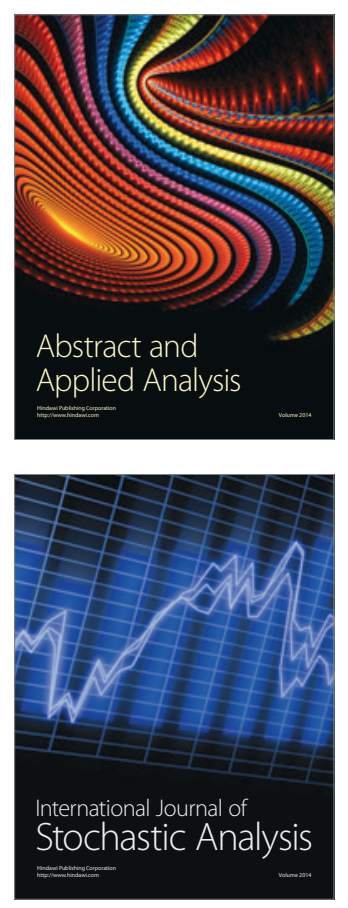

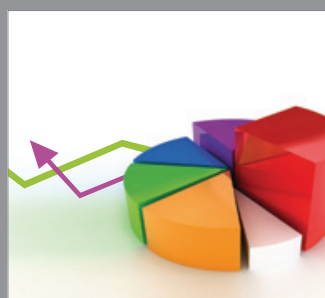

ournal of

Probability and Statistics

Promensencen
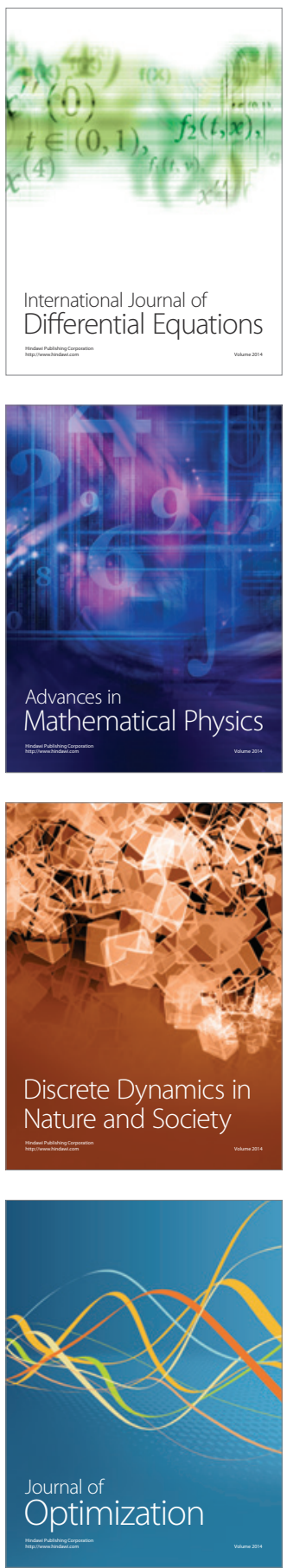